\title{
3D film viewer development and trial
}

\author{
Longbo Chen ${ }^{1, a}$, Jilin Guo ${ }^{1, a}$ and Qilin Yang ${ }^{1, a}$ \\ ${ }^{1}$ Gongqing Institute of Science and Technology, Jiangxi Province \\ a670365498@qq.com
}

\begin{abstract}
Keywords: 3D film viewer;3D imaging
Abstract. 3D film viewer is a kind of instrument that can display flat models that can display three-dimensional models. Its simple structure, clever design, and small size are suitable for large and small-format color or black-and-white flat pictures. You can view three-dimensional pictures consisting of perspective or axonometric measurements on the $\mathrm{H}$ surface, $\mathrm{V}$ surface, and positive axonometric screen. Observed stereo models can be measured directly with a transparent ruler or indirectly with a divider. If you swap the left and right pictures, you can see the symmetry of the three-dimensional model. If you rotate the picture by $180^{\circ}$, you can see the opposite side of the object. The 3D picture viewer has a wide range of uses in teaching, scientific research, production, medical treatment, surveying and mapping, urban and rural general planning, and military affairs. In particular, $3 \mathrm{D}$ viewers are used in place of expensive teaching models, building sand tables, product displays, etc. There will be major breakthroughs, which can save a lot of raw materials and storage and transportation costs, resulting in great economic and social benefits.
\end{abstract}

\section{Introduction}

Perspectives and axonometrics are three-dimensional, so they are usually called perspective views. However, they are the same as a three-dimensional floor plan. After all, they are all a plane figure. They are all projected from a projection center. That is, from the view of one eye, it is known that it is difficult to determine the depth of the near vision by the monocular vision, and only the binocular vision can be considered. For example, it is difficult to use one eye to point up two nibs and it is very easy to use the two eyes. Therefore, using this principle, the two eyes are used as two projection centers, and the objects are projected on the same plane (as shown in Figure 1). Show), get two perspective projections, this kind of projection is called double center projection. Now remove the space objects so that the two eyes are exactly at the two center positions of the projection pair. When the two eyes are directly visible to the original space with the naked eye (as shown in Figure 2), then, With visual function, we can see the true three-dimensional image formed by the convergence of the corresponding points of the two projections of the painted object. This observation method is called stereoscopic observation by naked eye. However, this kind of observation method is not easy to grasp for the people. After learning, it takes more effort and patience to practice. Now we have developed a simple and ingenious 3D film viewer that can effortlessly see black and white or color three-dimensional models with clear and vivid images and excellent results.

\section{Development of 3D film viewer}

The working principle of the 3D film viewer is actually to fit a pair of slightly different flat images correspondingly, so that the eyes can only see a picture they should see at the same time and get a true three-dimensional visual display of the three-dimensional model. The only way to achieve this principle is to use a flat mirror.

\section{The principle of instrument design using a flat mirror}

Let us first look at the imaging phenomenon of the plane mirror. As shown in Fig. 1(a), a light beam is emitted from any point 0 and is reflected by a plane mirror. According to the law of reflection, the extended line of the reflected light intersects with point $\mathrm{O}$. This $\mathrm{O}$ point is the virtual image of $\mathrm{O}$, 
located behind the mirror, and $\mathrm{O}$ point to the plane of the normal $\mathrm{ON}, \mathrm{ON}$ an oN. Therefore, the resulting image is the same size as the original, and the object is symmetrical to the mirror surface, as shown in Figure 1(b).

If there is an inverse map aL (inverse) of the left eye view before the mirror, there must be an opposite aL (inverse) aL (positive) virtual map behind the mirror, as shown in Figure 1(c).

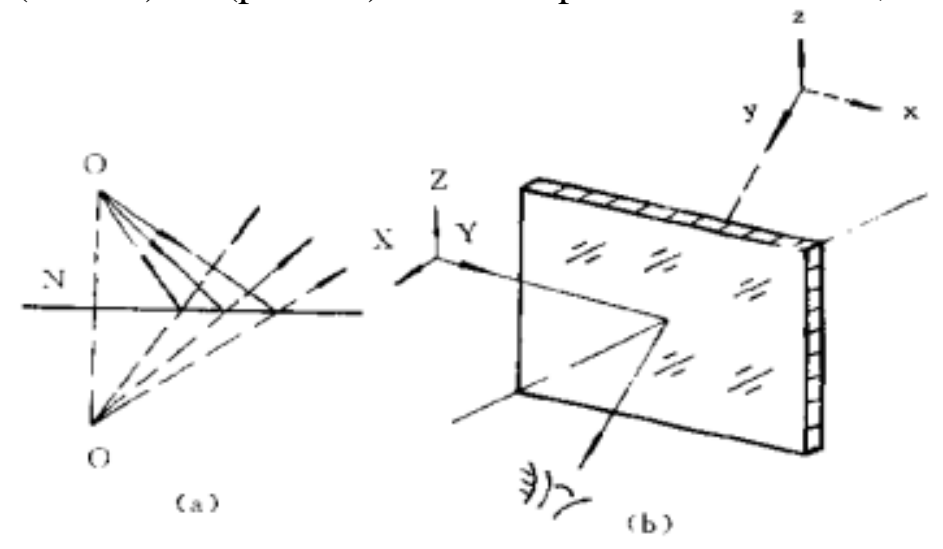

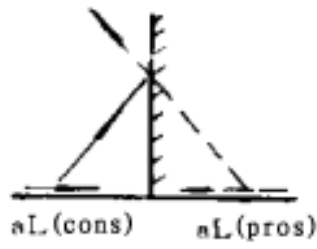

(c)

Figure 1 Reflection law of plane mirror

ConclusNow if the right eye view aR is placed on the corresponding position of the virtual map aL (positive), and the two eyes view the overlapping two plane projections at the same time across the mirror, the working principle of the above-mentioned instrument is correctly implemented. As shown in Figure 2(a), it is the simplest case, and the same results can be obtained in other cases of Figure 2. Visible, as long as the mirror is in the front and back of the picture plane angle flat surface, flat picture. $\mathrm{L}$ (the backhand image of the left eye view) can be combined with the reality of the planar image aR, and the stereoscopic model $\mathrm{A}(\mathrm{aL}, \mathrm{aR})$ is merged in the vision after the reflected virtual image.

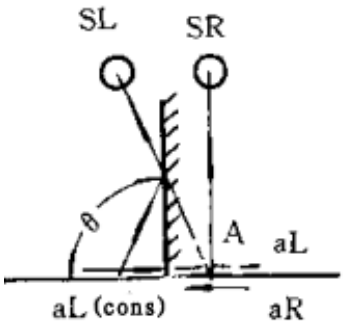

(a) $\theta=90^{\circ}$

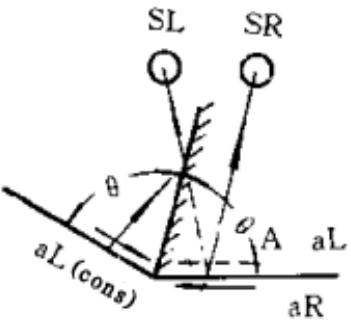

(b) $\begin{aligned} & \theta>60^{\circ} \\ &<90^{\circ}\end{aligned}$

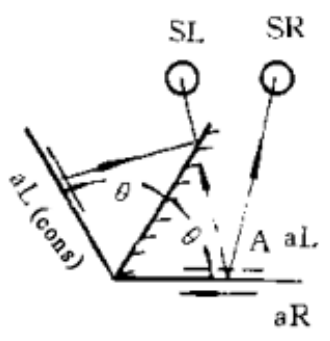

(c) $0=60^{\circ}$

Figure 2 Several schemes for viewing 3D pictures using a flat mirror

According to the normal human eyes, the normal cone range is about $60^{\circ}$, the stereoscopic image to be viewed is about $16 \mathrm{~K}$ book size, the stereoscopic model seen should be in the vicinity of the visible distance, and the instrument should be like a hardcover book after closing. For factors, we prefer to use Figure 2(c). Setting: the angle between the figure and the mirror $=60^{\circ}$, the height of the viewpoint $\mathrm{H}=$ $250 \mathrm{~mm}$, the interpupillary distance $\mathrm{B}=64 \mathrm{~mm}$, so as to make the principle design and its dimensions, as shown in Fig. 3.

\section{Matching a pair of flat pictures with film viewers}

Obtaining correct stereoscopic models from stereoscopic projection images and obtaining stereoscopic projection images from objects are two inverse systems that must be coordinated.

The drawing method of the stereogram matching this instrument, the size range of the three-dimensional model, and the size and position of the stereo projection are shown in FIG. 4 . The $\mathrm{H}$ surface can also be treated as a V surface, and the double centered point of view is the same. However, the instrument must be hung from a vertical position and viewed obliquely upwards or vice versa. The 
effect is the same, except that the direction of the cone in the legend has changed from the vertical axis to the positive vertical axis.

If the three-dimensional photograph matching this instrument is to be taken, if it is similar to the effect of the above three-dimensional image, only the wide-angle lens facing the picture ( $\mathrm{H}$ or $\mathrm{V})$ can be viewed on the edge, and the object distance and the optical axes of the two lenses can be achieved. Both the distance and the image print must be scaled up accordingly to meet the instrument's stereoscopic viewing requirements.

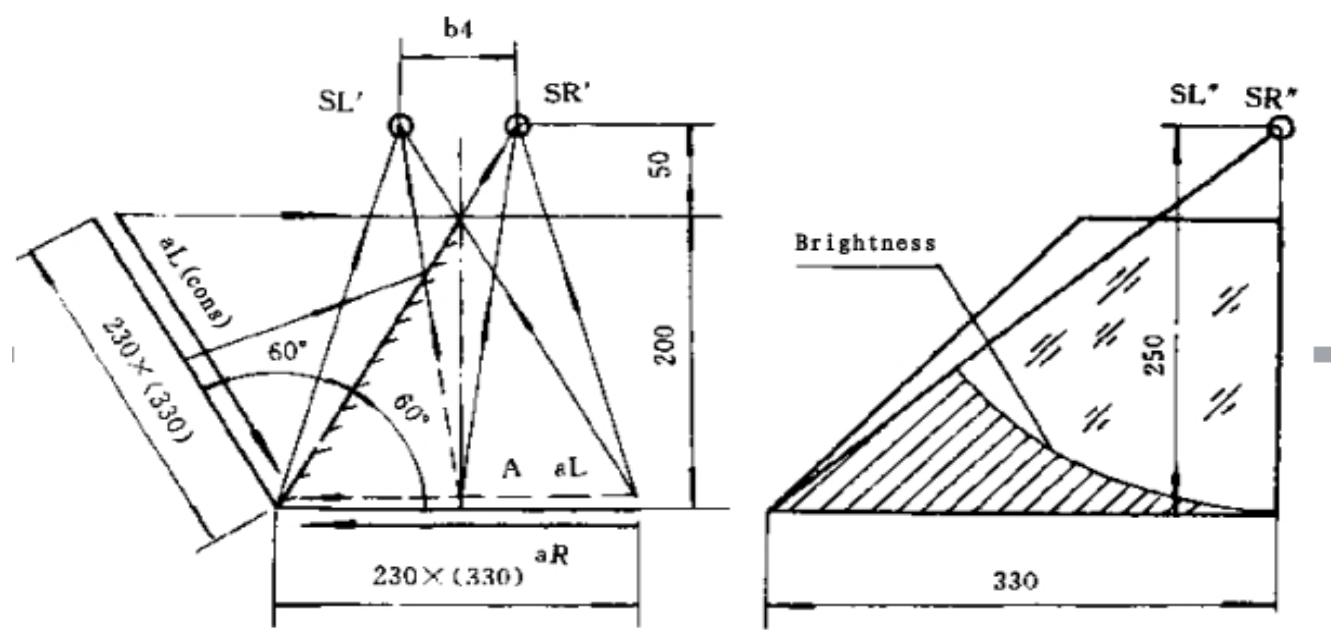

Figure 3 Instrument principle design for viewing stereoscopic images according to figure L(a)

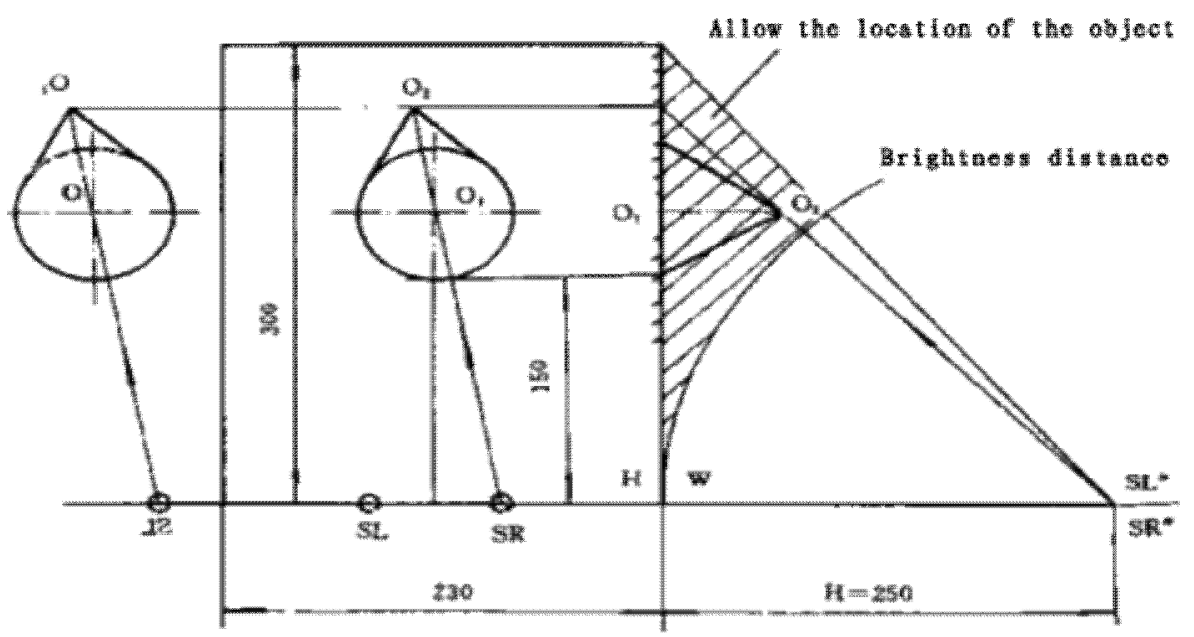

Figure 4 Drawing of a stereoscopic picture matching the instrument

\section{Structure Design of 3D Viewer}

The 3D film viewer is a box consisting of a cover frame and a bottom frame with the same shape. The two frames and the frame are connected with a hole and a shaft set, and can be opened or closed. The flat picture can slide back and forth in the two frames to adjust the position. The arm ensures that the frame is automatically positioned at the corners of the lid frame and the bottom frame at opening. One side of the frame opposite the cover frame is equipped with a plane mirror, and the upper end of the mirror is inserted with a guide plate. The two open eyes can guide the eyes to squint forward and downward in proper positions, but they can be used without skill, so as to allow more flexible viewing.

Figure 5 shows the structure of the 3D viewer. In addition to the lens and three screws, the remaining five parts are all made of injection molding, the assembly is simple, the angle between the bottom frame and the cover frame is $0^{\circ} \sim 180^{\circ}$, and the elasticity of the slider can be adjusted to make the plane mirror and the two frames clamp. Corners are randomly selected and automatically kept equal, generally about 60 is better. Hold the cover frame with your left hand and press the bottom frame with your right hand. You can switch as you like. The buckle is convenient for carrying when you close, and 
you can save pictures in the empty space inside the box. The structure design of this instrument has achieved five kinds of schemes such as (a), (b)and (c) shown in FIG. 2

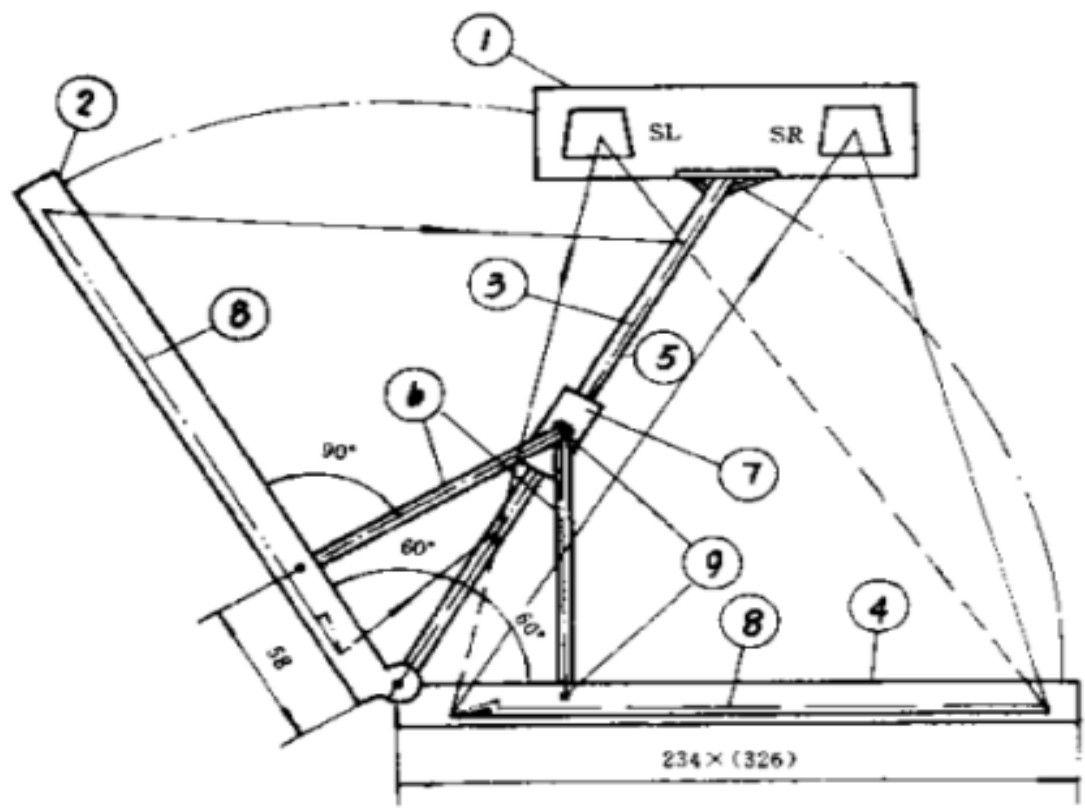

1.Guide panel 2. Cover frame 3. Plane mirror 4. Bottom frame 5. Frame 6. Brace arm 7. Slider 8. Plane picture 9. Screw

Figure 5 Structural design of 3D picture viewer

\section{Film viewer trial}

The 3D film viewer was tested in schools, factories, research institutes, hospitals and other units. There are university teachers, students, engineering technicians, workers, doctors, kindergarten teachers, etc., which are widely used in trial film viewers. Generally speaking, the effect is good. Everyone feels that two slightly different flat pictures are placed on the viewer. It is really God that we can clearly see the wonderful color and black and white optical models stand up. It has the following features compared to the current complementary color plan for viewing with red and green filter glasses:

(1) The stereoscopic model seen is more clear because it does not need to be viewed through the filter glasses and the graph lines are added in pairs.

(2) A color stereoscopic model can be vividly and realistically viewed as long as a colored floor plan is drawn and a color plane picture is taken. Therefore, it is also possible to imitate the color transparent plexiglass model.

(3) Since the plane picture is drawn separately and photographed at two places, there is no problem of discoloration of the lines and points where the corresponding lines overlap and non-corresponding lines intersect, and it is also possible to draw stereo images with any of the primary colors and photographs. The picture does not have a troublesome color matching problem, so it is convenient for the user to make a home-made (drawing or photographing) 3D picture temporarily.

(4) Since a single picture is an ordinary stereoscopic picture - perspective or axonometry also has a certain degree of three-dimensionality, and it also enhances the three-dimensionality when viewed with a single eye. Some people think that this can be satisfied, in fact, the difference is very far: because this is only a fake stereoscopic impression in the plane, only by looking at a pair of flat images with his eyes to see the true stereoscopic image of space. Therefore, people whose vision is generally normal can feel really" god" when they look at it, and the object really " stands up" on paper. Even people suffering from color blindness can see it. 
(5) Because the two pictures can be exchanged, the three-dimensional model seen before and after the exchange is a symmetry of the face, so it is possible to view the two similar left and right parts in the symmetrical part, and serve two purposes.

(6) Can be measured directly with a transparent ruler and protractor.

(7) When the head is translated back and forth, left and right, and up and down, the stereoscopic model seen will also be shaken back and forth, side-to-side, and height-varied.

(8) The instrument is compact in design, like a book, can be opened and closed, can save pictures, easy to carry, use and save.

(9) The bottom frame of the instrument lays flat on the desktop. You can see the H surface stereoscopic image. You can see the V surface stereoscopic image on the vertical wall. The bottom frame can look forward at an appropriate angle. You can see the axonometric or three-dimensional perspective. Looking forward at an appropriate angle can look up at the three-point perspective. If multiple instruments are properly arranged at the same time, special exhibitions can be made.

\section{Conclusion}

The successful development of the 3D film viewer has opened up a new field in 3D imaging technology. Its application prospect is very broad. In the teaching of various schools, especially in the correspondence and television teaching in remote areas, the exhibition instrument can greatly enhance the intuitiveness of the teaching field. It can replace most of the visual teaching aids and give students Bring great convenience. Through the observation and reflection on 3D pictures, it will be of great benefit to cultivate students' self-learning ability and enhance the understanding of content. The anatomy and medical use of the film viewer to observe the x-ray images can determine the size of the human internal organs, bone joints, and surgical conditions. In commodity exchanges, the use of film viewers and related stereoscopic images enables the appearance, shape, and configuration of large, medium, and small types of cartography.

The layout of the structure is clearly visible to the user. In urban and rural architectural planning, landscape planning, community design, interior decoration design, through the viewer to observe the corresponding picture, its presentation effect can completely replace the large body, expensive sandbox model. It also has good application value in art photography, such as the human body's 3D photographs. Observing through the film viewer can clearly show three-dimensional images and clear three-dimensional lines. Using the viewing instrument and related pictures, it is also possible to express intangible and unimaginable spatial shapes and patterns. If you can't see the invisible electromagnetic field, multi-dimensional space, etc. In short, the outlook in the future may become a world of 3D film viewers in all fields of image presentation and use of presentation effects. It will serve as a powerful tool for the information society as a supplement, extension and intensification of video and video recordings and books.

\section{References}

[1] Chen Siyu. Research on the design and application of naked eye 3D stereo vision [D]. Beijing University of Technology, 2016.

[2] Zhao Tianqi. Research on some key technologies for generating and displaying 3D content in the blink of an eye [D]. Beijing University of Posts and Telecommunications, 2015.

[3] Zhang Zhaoxing, simplified body view technology and its application (trial projection workshop trial teaching materials), 1988. 